\title{
Spectral Touching Points in Two-Dimensional Materials
}

\author{
Andrea $\mathrm{Wynn}^{\dagger}$ \\ Project advisor: Dr. Tracy Weyand
}

\begin{abstract}
A two-dimensional (2D) material is a crystalline material consisting of a single layer of atoms. These materials are used in many applications including photovoltaics, semiconductors, electrodes, and water purification. These materials' atomic structures can be represented as a discrete infinite periodic graph. Using Floquet-Bloch theory, the spectrum of the Schrödinger operator can be calculated on these infinite graphical representations by computing the eigenvalues of the magnetic flux Schrödinger operator on a fundamental domain for every possible value of magnetic flux. Previous researchers have conjectured a relationship between the special physical properties of one 2D material, graphene, and the Dirac conical points which appear in the spectrum of its Schrödinger operator. However, graphene was the only material studied with respect to these Dirac conical points. The existence of spectral touching points in different two-dimensional materials is proved, including muscovite, quartz, and transition metal oxides, under certain conditions on electric potential. The spectral touching points found in transition metal oxides are not the Dirac conical points found in graphene, but rather a previously unknown type of spectral touching point, named the mesa touching point, which appears in the Schrödinger operator for transition metal oxides under certain conditions.
\end{abstract}

1. Introduction. In recent years, graphene has gained significant popularity as a building material and energy storage medium with a wide variety of applications. One of graphene's most unique properties is its conductivity, which is enhanced by mass-less fermions that enable loss-less electron transfer across a graphene sheet (8). Due to its many very useful properties, graphene has been widely studied across many different disciplines.

One of these disciplines is spectral graph theory. This field encompasses the study of the spectra of operators which act upon graphs. For discrete graphs, such as those which are used throughout this work, operators that act upon the graphs are represented as matrices, and their spectra consist of the eigenvalues of the operator matrix. The main point of interest in this work is spectral touching points, which occur whenever an eigenvalue has multiplicity greater than one. Specifically, this work focuses on searching for Dirac conical points, which occur when spectral sheets form cone shapes near the point of contact.

Previous researchers (10) have conjectured a potential relation between the existence of Dirac conical points in the spectrum of the Schrödinger operator acting on graphene's 2D graph and the unique properties of graphene, although this has yet to be formally proven. Further research from Weyand (2), Berkolaiko (3) and Martin (8) found that any electric potential values satisfying certain symmetry conditions on graphene has these touching points.

This work builds upon previous research by investigating the structures of numerous 2D materials and proving the existence of spectral touching points in their Schrödinger operators. We prove that the same fundamental domain used in previous research can also be used to construct the infinite periodic graph representing the 2D materials bismuthene, germanene, silicene, stanene, and phosphorene, which proves the existence of spectral touching points in the spectrum of the Schrödinger operator for each of these materials under the same symmetry conditions as those found for graphene (2), (8), (3). The existence of a Dirac conical touching point in the spectrum of the Schrödinger operator acting on quartz for certain values of electric potential is proven. The existence of a previously unknown type of spectral touching point, named the mesa touching point, is also shown in the spectrum of the Schrödinger operator acting on transition metal oxides.

1.1. Outline. subsection 1.2 provides the relevant terms and their definitions which are used throughout this work. section 2 provides a detailed description of the main findings from this research. Specifically, novel findings are presented about a number of materials similar to graphene

\footnotetext{
${ }^{\dagger}$ Rose-Hulman Institute of Technology (wynna@rose-hulman.edu, https://andrea-00.github.io/).

${ }^{\ddagger}$ Rose-Hulman Institute of Technology (weyand@rose-hulman.edu)
} 
(bismuthene, germanene, silicene, stanene, and phosphorene); quartz; and transition metal oxides, and the existence of spectral touching points in each is proven. Finally, section 3 discusses the results and impact of this work, as well as outlining potential questions for continued research.

1.2. Terminology. A two-dimensional material, or $\mathbf{2 D}$ material, is a crystalline material consisting of a single layer of atoms. These materials' structure makes them uniquely suited for various real-world applications, especially electronics. A 2D material's atomic structure can be represented as an undirected discrete graph, which is defined as a set of vertices $V$ and a set of edges $E$ with each edge connecting exactly two vertices. The degree of a vertex is the number of edges which are connected to that particular vertex.

The discrete graph representation of a 2D material's structure is an example of an infinite periodic graph. The material has a repetitive (periodic) structure and, viewed on the atomic scale, can be considered to be infinite (if one is not very close to an outer boundary). This infinite periodic graph contains a finite subgraph that can be tessellated to produce the full infinite graph. A smallest finite subgraph of the infinite graph that satisfies the following properties (4) is called a fundamental domain:

1. The union of all shifts of the fundamental domain must cover the full infinite graph.

2. Different shifts of the fundamental domain must not share any vertices in common.

It is important to note that a fundamental domain for an infinite periodic graph is not necessarily unique; a single infinite periodic graph may have more than one fundamental domain. Vertices are defined as quasi-connected when they are not directly connected to each other by an edge in the chosen fundamental domain for an infinite periodic graph, but they are connected directly by an edge between different shifts of the fundamental domain. Figure 1 illustrates the idea of quasi-connected vertices.

Functions on discrete graphs are defined only on the vertices. Therefore, a function on this discrete graph can be represented as a vector storing the values of the function at each of the vertices of the graph. This also means that operators acting on the graph can be represented as matrices. The Schrödinger operator is an example of such an operator, and it is defined as follows:

$$
H^{A}=Q-A
$$

where

$$
Q_{u, v}= \begin{cases}q_{u} & \text { if } u=v \\ 0 & \text { otherwise }\end{cases}
$$

where $q_{u} \in \mathbb{R}$ represents the electric potential value at the vertex $u$, and $A$ is the adjacency matrix corresponding to the graph upon which the operator acts.

The graph representations of the $2 \mathrm{D}$ materials studied here are $\mathbb{Z}^{2}$-periodic, which means that two $\alpha$ variables are needed (namely $\alpha_{1}$ and $\alpha_{2}$ ) to represent the distance moved in two linearly independent directions, $\overrightarrow{x_{1}}$ and $\overrightarrow{x_{2}}$, in which the fundamental domain must be shifted to form the full infinite periodic graph. The spectrum of the Schrödinger operator acting upon the infinite graph is equivalent to the union over all $\alpha$ variables of the spectrum of the magnetic flux Schrödinger operator (which will be defined in subsection 2.1) acting on one copy of the fundamental domain. This spectrum equivalence is given by Floquet-Bloch theory.

A spectral sheet is a set of eigenvalues such that the $n^{t h}$ spectral sheet contains the $n^{\text {th }}$ eigenvalue of the magnetic flux Schrödinger operator for each combination of values of $\alpha_{1}$ and $\alpha_{2}$. A spectral touching point occurs when two spectral sheets from the spectrum of the Schrödinger operator touch. To prove that such a touching point does exist, one must find a duplicated eigenvalue in the spectrum of the magnetic flux Schrödinger operator that occurs for a specific value of $\alpha_{1}$ and $\alpha_{2}$ which also corresponds to two or more linearly independent eigenvectors. A Dirac conical point is a special class of spectral touching point where the spectral surfaces of the two eigenvalues form cones near the point of contact. 


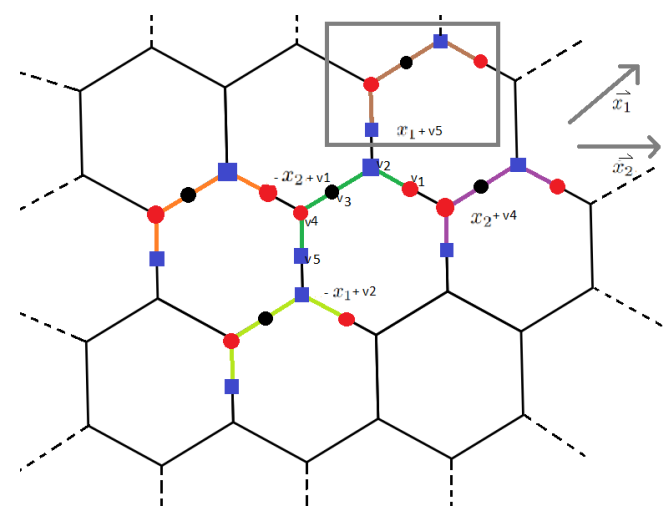

Figure 1: The full infinite periodic graph representing graphene's 2D structure, with five iterations of the chosen fundamental domain (one iteration enclosed by a gray box). Quasi-connected pairs are denoted with the same shape and color. Each shift of the fundamental domain with vertices $v_{1}$, $v_{2}, v_{3}, v_{4}, v_{5}$ in the positive and negative $\overrightarrow{x_{1}}$ and $\overrightarrow{x_{2}}$ directions are indicated; for instance, the blue vertex in the gray bounding box is the vertex 5 shifted by $\overrightarrow{x_{1}}$. This demonstrates how iterations of the chosen fundamental domain are tiled together to form the full infinite graph. The vectors $\overrightarrow{x_{1}}$ and $\overrightarrow{x_{2}}$ are shown at the top right. Note that vertex $v_{3}$ does not have a quasi-connected pair, and therefore is the only vertex of its color and shape in that fundamental domain.

\section{Main Results.}

2.1. Previous Results: Graphene. We will first study the structure and properties of graphene, as well as a number of other materials whose structures resemble graphene's hexagonal 2D lattice structure. The vertices in this graph each represent a carbon atom, and the edges connecting them represent covalent bonds. Figure 1 shows a section of the infinite periodic graph representing graphene's 2D structure.

We consider the fundamental domain shown in Figure 1 for this infinite periodic graph (8), (2). Quasi-connected vertex pairs represented as pairs of vertices with matching shapes and colors, overlaid on the infinite periodic graph representation of graphene. Note that vertex $v_{3}$ does not have a quasi-connected pair and therefore is the only vertex of its color.

Figure 1 displays five separate iterations of the chosen fundamental domain, overlaid on the infinite periodic graph. This demonstrates how the fundamental domain can be tiled together to produce the full infinite graph by connecting the quasi-connected vertices across neighboring iterations of the fundamental domain. The vectors $\overrightarrow{x_{1}}$ and $\overrightarrow{x_{2}}$ are also shown.

Let $\overrightarrow{x_{1}}$ and $\overrightarrow{x_{2}}$ be two vectors used to tile the entire infinite graph, as shown in Figure 1. Let $\gamma=\left(\gamma_{1}, \gamma_{2}\right)$ represent how far a fundamental domain is moved in each direction $\overrightarrow{x_{1}}$ or $\overrightarrow{x_{2}}$ to get from any point $y$ to any other point $z$ in the graph (i.e. $z=\gamma_{1} \overrightarrow{x_{1}}+\gamma_{2} \overrightarrow{x_{2}}+y$ ). Let $\alpha_{1}$ and $\alpha_{2}$ be variables (numbers) ranging over $[-\pi, \pi]$, with $\vec{\alpha}=\left(\alpha_{1}, \alpha_{2}\right)$. Let $c=\gamma_{1} \alpha_{1}+\gamma_{2} \alpha_{2}$ be the shift between one point and another. The magnetic flux Schrödinger operator can be found using the following process (2):

1. Find a fundamental domain and label all its vertices. Define the set of vertices $V=$ $\left\{v_{1}, v_{2}, \ldots, v_{n}\right\}$ where $n$ is the total number of vertices in the fundamental domain.

2. Define two linearly independent vectors, $\overrightarrow{x_{1}}$ and $\overrightarrow{x_{2}}$, pointing in the directions in which the fundamental domain must be shifted to tile the infinite graph. See Figure 1.

3. For each vertex $v_{i}, i \in\{1,2, \ldots, n\}$, let $V_{i}$ be the set of all vertices connected to $v_{i}$, and let $f_{i}$ denote the function value on the vertex $v_{i}$. The corresponding eigenvalue problem to solve is $\lambda f_{i}=q_{i} f_{i}-\sum_{v_{k} \in V_{i}} f_{k}$ where $v_{k} \neq v_{i}, v_{k}$ is adjacent to $v_{i}$ and $q_{i}$ represents the value of the electric potential at vertex $v_{i}$. If $v_{k}$ (attached to $v_{i}$ ) belongs to a different, shifted copy of the fundamental domain, include the coefficient $e^{i c}$. 
4. Write these $n$ equations into the matrix form $H^{\vec{\alpha}} f=\lambda f$ to turn this into an eigenvalue/eigenvector problem.

Mathematically, this is the first step of Floquet-Bloch theory. The union is then taken over all values of $\alpha_{1}$ and $\alpha_{2}$ to study the spectrum of the Schrödinger operator $H^{A}$ on the infinite periodic graph by analyzing the magnetic flux Schrödinger operator $H^{\vec{\alpha}}$ acting on the chosen fundamental domain for all values of $\alpha$ (2). Additionally, by Euler's formula, since $e^{i c}=\cos (c)+i \sin (c)$, restricting the range of $\alpha_{1}$ and $\alpha_{2}$ to $[-\pi, \pi]$ will give every possible value of this complex number, meaning that only $\alpha$ values within this range need to be studied.

Following the procedure above, the resulting matrix $H^{\vec{\alpha}}$, shown below, is the magnetic flux Schrödinger operator acting on a fundamental domain of the graph representing the atomic structure of graphene:

$$
H^{\vec{\alpha}}=\left[\begin{array}{ccccc}
q_{1} & -1 & 0 & -e^{i \alpha_{1}} & 0 \\
-1 & q_{2} & -1 & 0 & -e^{i \alpha_{2}} \\
0 & -1 & q_{3} & -1 & 0 \\
-e^{-i \alpha_{1}} & 0 & -1 & q_{4} & -1 \\
0 & -e^{-i \alpha_{2}} & 0 & -1 & q_{5}
\end{array}\right]
$$

Note that we want to compute the spectrum of $H^{A}$ as defined earlier, but since $H^{A}$ acts upon an infinite graph, $H^{A}$ would be an infinite matrix. Floquet-Bloch theory tells us that the infinite spectrum of $H^{A}$ can be found by determining the finite spectrum of $H^{\vec{\alpha}}$ on a fundamental domain for all possible values of alpha.

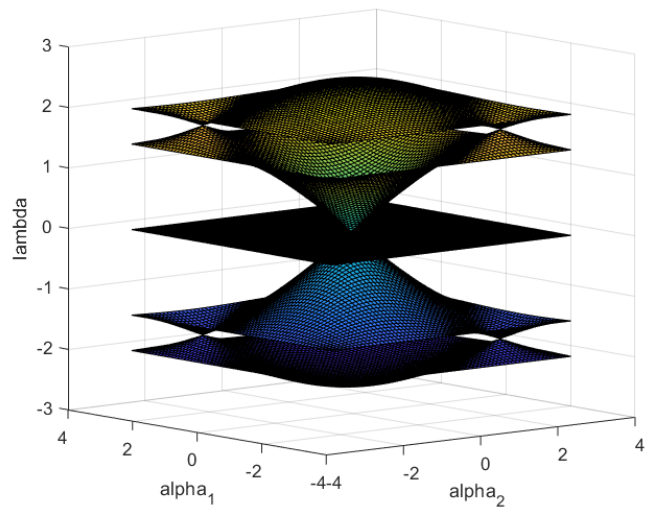

Figure 2: The plot of the spectrum of graphene. The $\mathrm{x}$ and $\mathrm{y}$ axes represent the values of $\alpha_{1}$ and $\alpha_{2}$ respectively, ranging from $-\pi$ to $\pi$, and the $\mathrm{z}$ (vertical) axis represents the eigenvalue.

Dirac conical points form in the spectrum of graphene for all values of electric potential, as long as certain symmetry conditions are maintained on the electric potential values at each vertex of the graph (8). Figure 2 shows the plot of the spectrum of the magnetic flux Schrödinger operator acting on the fundamental domain, with respect to $\alpha_{1}$ and $\alpha_{2}$. The Dirac conical points are clearly visible in the spectrum plot (Figure 2) between sheets 2 and 4 with $\lambda=0$ and $\alpha_{1}=\alpha_{2}=0$; the symmetry conditions are enforced by setting $q_{4}=q_{2}$ and $q_{5}=q_{1}$ with all $q \in \mathbb{R}$.

\subsection{Materials Similar to Graphene.}

Theorem 2.1 (Dirac Conical Points in Graphene-like Materials). For all values of electric potential, as long as certain symmetry conditions are maintained (8), the spectrum of the Schrödinger operator acting on the discrete graph representation of muscovite, bismuthene, germanene, silicene, stanene, and phosphorene have Dirac conical points.

Because this property of the spectrum of graphene's discrete infinite periodic graph representation was proven for the chosen fundamental domain, these results can also be generalized to any 
other 2D material which shares the same infinite periodic graph as graphene, provided that the same fundamental domain is chosen and the same symmetry conditions (8) are applied.

There are many other 2D materials which share the same 2D hexagonal lattice structure: bismuthene, germanene, silicene, stanene, and phosphorene. These elements each have interesting properties discussed below, but all share the same infinite periodic graph representation of their atomic structure with graphene.

Bismuthene consists of a single atomic layer of bismuth atoms, arranged in a hexagonal structure just like graphene. Bismuthene is more stable than many other 2D materials, and unlike graphene, bismuthene is a room-temperature topological insulator and not a conductor. Bismuthene is used for many electronic applications, including spintronic devices, computing and data transmission, quantum spin Hall insulating materials, and saturable absorbers (5).

Germanene is a newer member of the graphene family which also shares a similar hexagonal lattice structure of germanium atoms. Germanene is the ideal material for exhibiting the quantum spin Hall effect at experimentally feasible temperatures, and it can be used in transistors without major differences between it and silicene (1).

Silicene also has a hexagonal lattice structure, though it is slightly buckled and composed of silicon atoms, whereas graphene's hexagonal lattice structure is planar. Silicene's electronic structure is also appears very promising for a variety of new applications, including quantum sensing and energy devices. Additionally, some researchers suggest that silicene may be highly useful in transitioning to ubiquitous semiconductor technology (9).

Stanene, composed of tin atoms, is best known for its potential for superconductivity. A single sheet of this material is predicted to host the quantum spin Hall effect at room temperature, which would also make it ideal for further studying this effect. Recent research on stanene indicates that it may provide the basis for novel strategies for constructing $2 \mathrm{D}$ topological superconductors (7).

Phosphorene, a 2D material composed of phosphorous, has begun to gain greater attention from the scientific world in recent years, following partially from the discovery of graphene. Phosphorene has enormous potential for applications in electronics, energy and infrastructure, and is also highly similar to graphene and transition metal dichalcogenides. It has additional opportunities for applications in nano-electronics, optoelectronics, energy conversion and storage, and sensors, which also arise from its exceptional properties resembling those of graphene (6).

Because these materials share the same 2D infinite periodic graph representation as graphene, the Dirac conical points occurring in graphene must also occur in these materials, as explained above. The equivalence of these materials' structures to graphene rests upon 2 assertions: the 2D structure must be represented as a two-dimensional discrete graph, with vertices of the graph defined at the points where atoms exist in the materials; and any differences in the edge lengths of the graphs, corresponding to the distance between the atoms, are not considered, because the discrete graph is an approximation of the true continuous case and electric potential is defined only at the vertices of the graph. Edge length is not accounted for in the discrete magnetic flux Schrödinger operator for this reason.

We will now consider another material, muscovite (also known as phyllosilicate or common mica). Muscovite is composed of aluminum and potassium atoms, whereas graphene is composed of carbon atoms; however, since our model does not treat different types of atoms differently, this difference between muscovite and graphene is considered irrelevant within this simplification. Muscovite also has a hexagonal lattice structure, but with one notable difference: it has an additional atom on each of the edges of the lattice. When translating this to the $2 \mathrm{D}$ graph, this results in additional vertices appearing on these edges, as shown in Figure 3.

However, despite the addition of these extra vertices, the same fundamental domain chosen for graphene still works for muscovite. The reason why the same fundamental domain can still be used, despite the additional atoms in muscovite's structure, is related to the idea of discrete and metric graphs. In the context of 2D materials, the discrete graph approximates a quantum graph, or metric graph, which models a portion of the atomic structure of a material. Figure 4 provides 


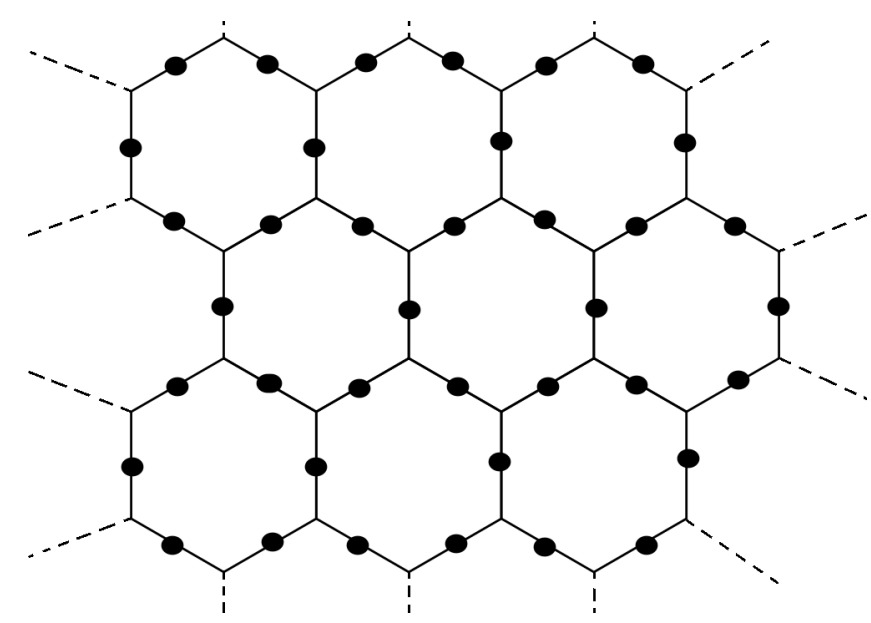

Figure 3: The infinite periodic graph representation of muscovite's 2D structure, with vertices chosen at the points where atoms exist in muscovite's structure.

an example of a metric vs discrete graph. Adding extra vertices on a discrete graph provides more information because functions are defined only at the vertices; however, functions acting on a quantum (continuous) graph are defined everywhere along the edges and vertices of the graph.
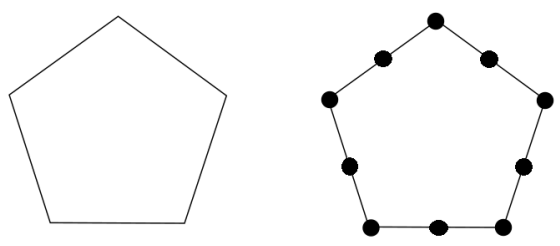

Figure 4: Contrast between a discrete vs metric graph. The metric (continuous) graph is on the left and the discrete graph is on the right, and the vertices of the discrete graph are indicated with solid circles.

The electric potential is only defined on the vertices of the discrete graph, and the discrete graph is an approximation of the true metric graph. Therefore, degree 2 vertices may be added or removed from the graph without changing the structure of the graph, as these vertices do not encode additional information about the electric potential values within the atomic structure that cannot be deduced from the electric potential at other vertices. For this reason, graphene and muscovite are mathematically identical with respect to their $2 \mathrm{D}$ graphs. This means that muscovite has the same mathematical properties, in this case Dirac conical touching points, as graphene despite its additional atoms.

Therefore, bismuthene, germanene, silicene, phosphorene, and muscovite all have 2D hexagonal lattice structures which can be constructed using the same fundamental domain that was studied for graphene by previous researchers (8), (2). Because they can be constructed using the same fundamental domain, we can conclude that for these materials, Dirac conical points form in their spectra for all values of electric potential, as long as certain symmetry conditions (8) are maintained on the graph.

2.3. Quartz. We will now study the structure and properties of quartz. Quartz is composed of silicon and oxygen atoms, and it has an atomic structure which is clearly distinct from that of graphene, with some hexagonal elements arranged around star-shaped figures, as shown in Figure 5 .

We will first simplify this graph by removing all vertices of degree 2 . We may do this without 


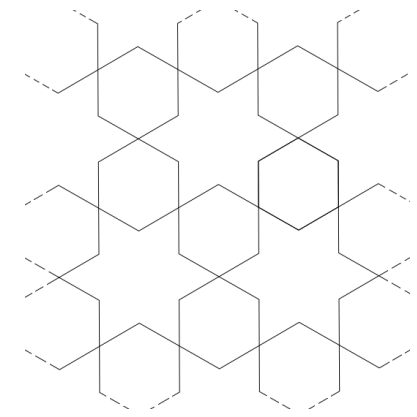

Figure 5: The infinite periodic graph representation of quartz' 2D atomic structure.

changing the structure of the graph for the same reason as explained in the previous section with the example of adding degree 2 vertices to create muscovite's graph from graphene's. This results in the simplified infinite periodic graph for quartz, shown in Figure 6. The chosen fundamental domain for this simplified infinite graph is shown in Figure 7.

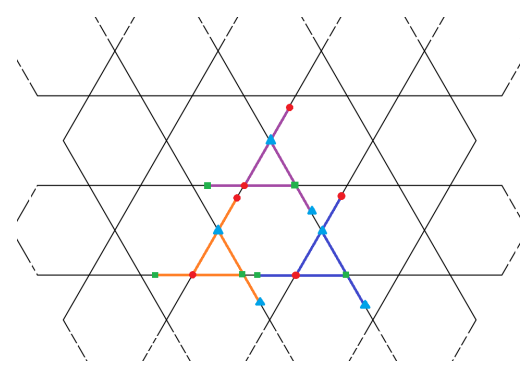

Figure 6: The simplified infinite periodic graph representation of quartz' 2D atomic structure, with all degree 2 vertices removed.

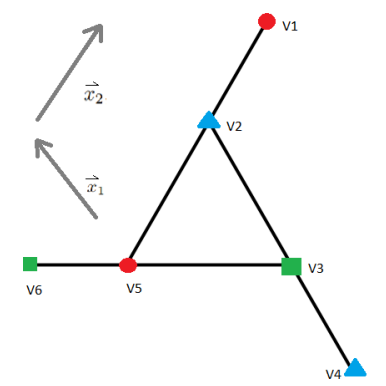

Figure 7: The chosen fundamental domain for the simplified infinite periodic graph for quartz. Quasi-connected pairs are denoted with the same shape and color, and $\overrightarrow{x_{1}}$ and $\overrightarrow{x_{2}}$ are indicated on the left.

Theorem 2.2 (Dirac Conical Points in Quartz). For certain values of electric potential, the spectrum of the Schrödinger operator acting on the discrete graph representation of quartz has Dirac conical points.

Using the procedure outlined in subsection 2.1, we obtain the magnetic flux Schrödinger operator acting on the chosen fundamental domain of quartz: 


$$
H^{\vec{\alpha}}=\left[\begin{array}{cccccc}
q_{1} & -1 & 0 & 0 & -e^{i \alpha_{2}} & 0 \\
-1 & q_{2} & -1 & -e^{i \alpha_{1}} & -1 & 0 \\
0 & -1 & q_{3} & -1 & -1 & -e^{i\left(\alpha_{2}-\alpha_{1}\right)} \\
0 & -e^{-i \alpha_{1}} & -1 & q_{4} & 0 & 0 \\
-e^{-i \alpha_{2}} & -1 & -1 & 0 & q_{5} & -1 \\
0 & 0 & -e^{i\left(\alpha_{1}-\alpha_{2}\right)} & 0 & -1 & q_{6}
\end{array}\right]
$$

where $q_{i} \in \mathbb{R}$ represents the electric potential at vertex $v_{i}$ of the fundamental domain. Vertex numbers on the fundamental domain are indicated in Figure 7.

Let $q_{2}=q_{3}=q_{5}=0$ and $q_{1}=q_{4}=q_{6}=1$. Additionally, let $\alpha_{1}=0$ and $\alpha_{2}=0$. We will prove that the magnetic flux Schrödinger operator with these values of electric potential has a spectral touching point. In this case:

$$
H^{\overrightarrow{0}}=\left[\begin{array}{cccccc}
1 & -1 & 0 & 0 & -1 & 0 \\
-1 & 0 & -1 & -1 & -1 & 0 \\
0 & -1 & 0 & -1 & -1 & -1 \\
0 & -1 & -1 & 1 & 0 & 0 \\
-1 & -1 & -1 & 0 & 0 & -1 \\
0 & 0 & -1 & 0 & -1 & 1
\end{array}\right]
$$

Define the following vectors:

$$
\overrightarrow{v_{1}}=\left[\begin{array}{c}
-1 \\
1 \\
-1 \\
0 \\
0 \\
1
\end{array}\right], \overrightarrow{v_{2}}=\left[\begin{array}{c}
-2 \\
1 \\
-1 \\
0 \\
1 \\
0
\end{array}\right], \text { and } \overrightarrow{v_{3}}=\left[\begin{array}{c}
1 \\
-1 \\
0 \\
1 \\
0 \\
0
\end{array}\right] \text {. }
$$

Observe that $H \overrightarrow{v_{1}}=2 \overrightarrow{v_{1}}, H \overrightarrow{v_{2}}=2 \overrightarrow{v_{2}}$, and $H \overrightarrow{v_{3}}=2 \overrightarrow{v_{3}}$. Thus, $\lambda=2$ is an eigenvalue with multiplicity 3 for the magnetic flux Schrödinger operator $H$, and $\overrightarrow{v_{1}}, \overrightarrow{v_{2}}$, and $\overrightarrow{v_{3}}$ are its corresponding eigenvectors. Additionally, the last elements of $\overrightarrow{v_{2}}$ and $\overrightarrow{v_{3}}$ are 0 , and the last element of $\overrightarrow{v_{1}}$ is nonzero; the $5^{\text {th }}$ elements of $\overrightarrow{v_{1}}$ and $\overrightarrow{v_{3}}$ are 0 , and the $5^{\text {th }}$ element of $\overrightarrow{v_{2}}$ is nonzero; and the $4^{\text {th }}$ elements of $\overrightarrow{v_{1}}$ and $\overrightarrow{v_{2}}$ are 0 , and the $4^{\text {th }}$ element of $\overrightarrow{v_{3}}$ is nonzero. Therefore, $\overrightarrow{v_{1}}, \overrightarrow{v_{2}}$, and $\overrightarrow{v_{3}}$ are linearly independent, so the Schrödinger operator has a spectral touching point. Thus, we have proven that there exists a spectral touching point for the specified $q$ values and at the specified $\alpha$ values of the magnetic flux Schrödinger operator acting upon the chosen fundamental domain of quartz.

Figure 8 shows the plot of the spectrum of quartz, where a Dirac conical spectral touching point forms between the top and third spectral sheets. This class of touching point is characterized by conical shaped spectral sheets near the point of contact.

2.4. Transition Metal Oxides. We will now study the structure and properties of transition metal oxides. Transition metal oxides have a complex 2-dimensional structure, which includes vertices of degree 6 and 3, as shown in Figure 9.

Again, we begin by defining a fundamental domain for this infinite periodic graph. Figure 10 shows the element's chosen fundamental domain, with quasi-connected vertices represented with matching shapes and colors. Figure 11 shows the chosen fundamental domain tiled into the infinite graph representing transition metal oxides' structure.

Theorem 2.3 (Dirac Conical Points in Transition Metal Oxides). For certain values of electric potential, the spectrum of the Schrödinger operator acting on the discrete graph representation of transition metal oxides has mesa touching points.

Using the procedure outlined in subsection 2.1, and $\overrightarrow{x_{1}}$ and $\overrightarrow{x_{2}}$ as defined in Figure 11, we obtain the magnetic flux Schrödinger operator acting on the chosen fundamental domain of transition metal 


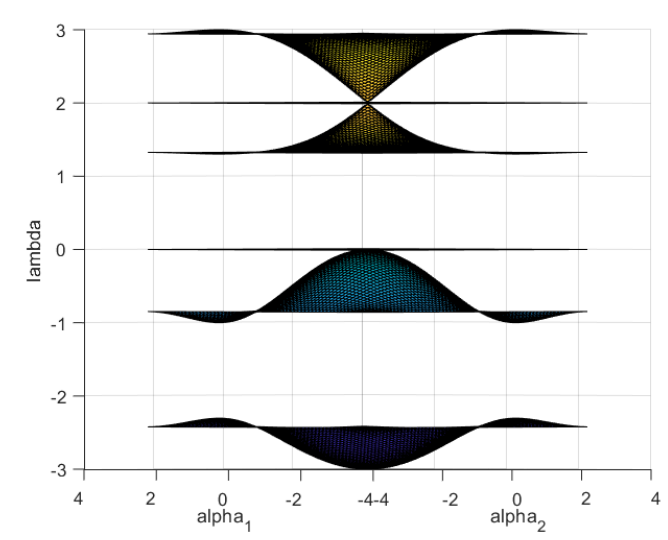

Figure 8: A plot of the spectrum of the Schrödinger operator acting on a discrete graph representation of quartz for a specific combination of electric potential values. There is a Dirac conical touching point as can be seen by the conical shape of the spectral sheets near the point of contact.

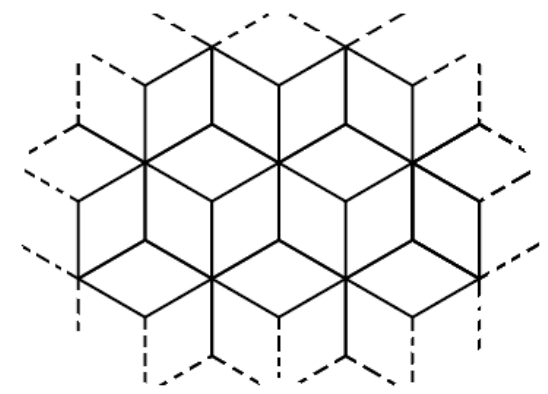

Figure 9: The infinite periodic graph representation of transition metal oxides' 2D atomic structure.

oxides:

$$
H^{\vec{\alpha}}=\left[\begin{array}{ccccccc}
q_{1} & 0 & -e^{i \alpha_{1}} & 0 & -e^{i \alpha_{2}} & 0 & -1 \\
0 & q_{2} & 0 & -e^{i \alpha_{2}} & 0 & -e^{i\left(\alpha_{2}-\alpha_{1}\right)} & -1 \\
-e^{-i \alpha_{1}} & 0 & q_{3} & 0 & 0 & 0 & -1 \\
0 & -e^{-i \alpha_{2}} & 0 & q_{4} & 0 & 0 & -1 \\
-e^{-i \alpha_{2}} & 0 & 0 & 0 & q_{5} & 0 & -1 \\
0 & -e^{i\left(\alpha_{1}-\alpha_{2}\right)} & 0 & 0 & 0 & q_{6} & -1 \\
-1 & -1 & -1 & -1 & -1 & -1 & q_{7}
\end{array}\right]
$$

where $q_{i} \in \mathbb{R}$ represents the electric potential at vertex $v_{i}$ of the fundamental domain. Vertex numbers on the fundamental domain are indicated in Figure 10.

Let $q_{1}=q_{2}=q_{4}=q_{6}=0$ and $q_{3}=q_{5}=q_{7}=1$. Additionally, let $\alpha_{1}=0$ and $\alpha_{2}=$ $\pi-\cos ^{-1}(3 / 8)$. As shown below, the magnetic flux Schrödinger operator with these values of electric potential has a duplicated eigenvalue: 


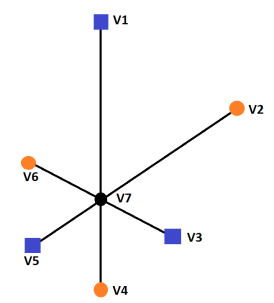

Figure 10: The chosen fundamental domain for transition metal oxides.

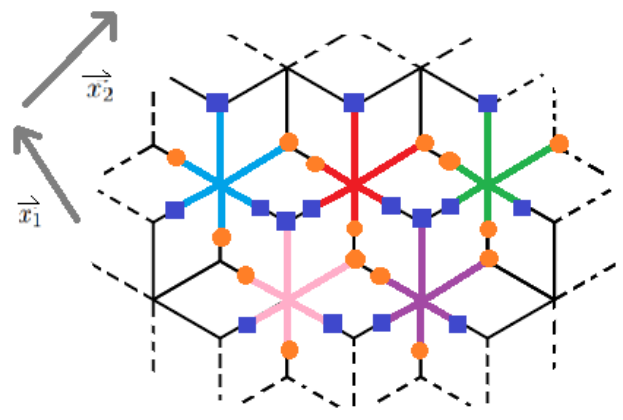

Figure 11: Multiple iterations of the chosen fundamental domain tiled together to form the full infinite graph representation of transition metal oxides. Quasi-connected pairs are denoted with the same shape and color, and $\overrightarrow{x_{1}}$ and $\overrightarrow{x_{2}}$ are indicated on the left.

$H^{\vec{\alpha}}=\left[\begin{array}{ccccccc}0 & 0 & -1 & 0 & -e^{i\left(\pi-\cos ^{-1}(3 / 8)\right)} & 0 & -1 \\ 0 & 0 & 0 & -e^{i\left(\pi-\cos ^{-1}(3 / 8)\right)} & 0 & -e^{i\left(\pi-\cos ^{-1}(3 / 8)\right)} & -1 \\ -1 & 0 & 1 & 0 & 0 & 0 & -1 \\ 0 & -e^{-i\left(\pi-\cos ^{-1}(3 / 8)\right)} & 0 & 0 & 0 & 0 & -1 \\ -e^{-i\left(\pi-\cos ^{-1}(3 / 8)\right)} & 0 & 0 & 0 & 1 & 0 & -1 \\ 0 & -e^{-i\left(\pi-\cos ^{-1}(3 / 8)\right)} & 0 & 0 & 0 & 0 & -1 \\ -1 & -1 & -1 & -1 & -1 & -1 & 1\end{array}\right]$

Define the following vectors:

$$
\overrightarrow{v_{1}}=\left[\begin{array}{c}
0 \\
0 \\
0 \\
-1 \\
0 \\
1 \\
0
\end{array}\right] \text { and } \overrightarrow{v_{2}}=\left[\begin{array}{c}
-\frac{13-i \sqrt{55}}{16} \\
\frac{46+6 i \sqrt{55}}{8(i \sqrt{55}-3)} \\
\frac{3-i \sqrt{55}}{16} \\
-\frac{8}{i \sqrt{55}-3} \\
\frac{i \sqrt{55}-19}{2(i \sqrt{55}-3)} \\
0 \\
1
\end{array}\right]
$$

Observe that $H^{\vec{v}} \overrightarrow{v_{1}}=\overrightarrow{0}$ and $H^{\vec{v}} \overrightarrow{v_{2}}=\overrightarrow{0}$. Thus, $\lambda=0$ represents the duplicated eigenvalue for the magnetic flux Schrödinger operator $H$, and $\overrightarrow{v_{1}}$ and $\overrightarrow{v_{2}}$ are its corresponding eigenvectors. Additionally, the $6^{\text {th }}$ element of $\overrightarrow{v_{1}}$ is nonzero, and the $6^{\text {th }}$ element of $\overrightarrow{v_{2}}$ is 0 , so $\overrightarrow{v_{1}}$ and $\overrightarrow{v_{2}}$ must be linearly independent. Therefore, since the magnetic flux Schrödinger operator has a duplicated eigenvalue, the Schrödinger operator has a spectral touching point. Hence, there exists a spectral touching point for the specified $q$ values and at the specified $\alpha$ values of the magnetic flux Schrödinger operator acting upon the chosen fundamental domain of transition metal oxides. 


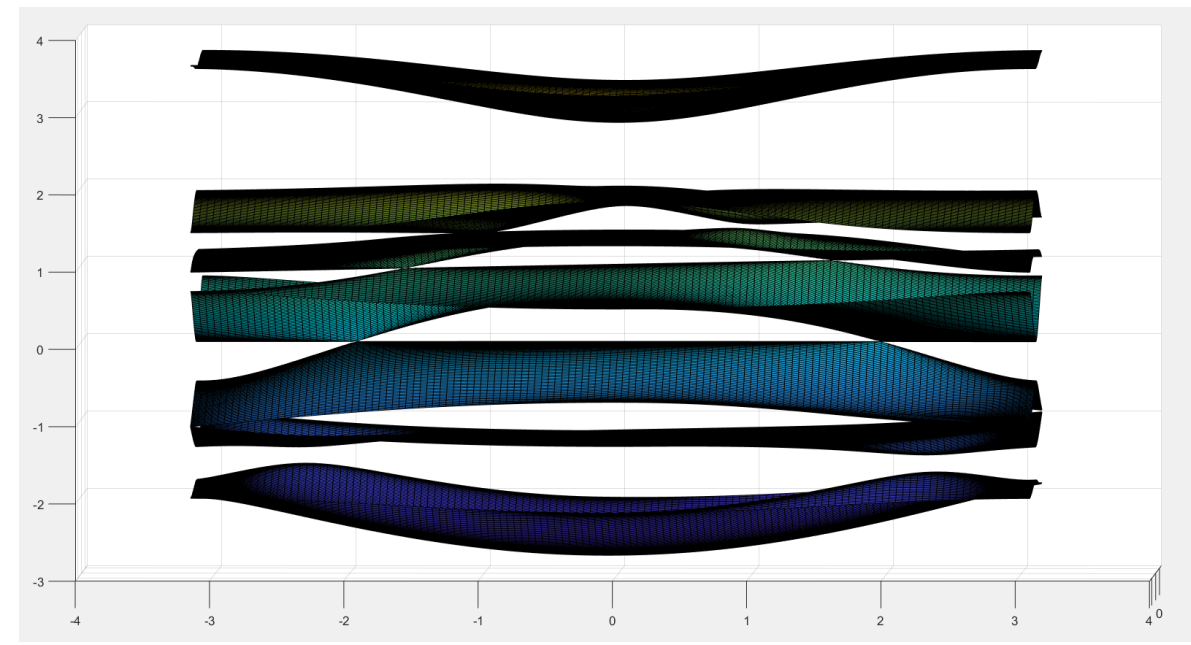

Figure 12: A plot of the spectrum for a specific combination of electric potential values. These are mesa touching points and not Dirac conical touching points, due to the flat region between the two touching points that occur between a pair of spectral sheets.

Figure 12 shows the plot of the spectrum of the Schrödinger operator acting on the discrete graph representation of transition metal oxides, where two touching points form between the $2^{\text {nd }}$ and $3^{\text {rd }}$ sheets, as well as between the $3^{\text {rd }}$ and $4^{\text {th }}$ sheets. The touching point at $\lambda=0$ between the third and fourth sheets was proven above.

As can be seen from the plot, these cannot be classified as Dirac conical touching points due to the flat region between the touching points which prevents conical shapes from forming near the point of contact. These touching points are a new type of spectral touching point which have not yet been observed in this field of study. This gives rise to a new class of spectral touching points, named mesa touching points, after the flat-topped hills that they resemble.

Theorem 2.4 (Mesa Touching Points Definition). Mesa touching points are characterized by a pair of spectral touching points that occur between the same two spectral sheets, with a flat region on the lower sheet between the points of contact.

3. Conclusions. There are some $2 \mathrm{D}$ materials, in addition to graphene, that also possess spectral touching points, including a new type of touching point that hasn't been studied before, namely mesa touching points. This sets a precedent for further investigating the conjectured relationship between Dirac conical points and the special physical properties of graphene as a 2D material.

In the future, it would be interesting to continue investigating some additional questions related to this work, including generalizing some of the results found here. For example, the following questions may prompt further study in this field:

- For what materials, and for what values of electric potential, are there touching points where $\alpha_{1}$ and $\alpha_{2}$ are both nonzero?

- What aspect of transition metal oxides' infinite periodic graph representation or atomic structure caused the occurrence of mesa touching points?

- Are there any other materials which have mesa touching points under certain conditions?

- Under what symmetry conditions on the electric potential values $q_{i}$ of the fundamental domain do spectral touching points occur for transition metal oxides and quartz?

It would also be interesting to study the physical properties of other materials with spectral touching points and see whether there exists any relationship with physical properties similar to those of graphene. Quartz and muscovite would be especially interesting to study, since those have a distinct physical structure from graphene but still have Dirac conical touching points, at least under certain conditions. It would also be interesting to investigate potential implications of mesa 
touching points for physical material properties, and to see whether these touching points occur in other materials as well.

Acknowledgments. First, the author would like to acknowledge her advisor, Dr. Tracy Weyand, who helped guide the author through her first research project in mathematics and who was always supportive and encouraging throughout the project. Dr. Weyand's patience, understanding, and willingness to try new things served as an inspiration and helped the author grow as a researcher. The author would like to thank her academic advisor, Ms. Amanda Stouder, for the suggestion to try research in the mathematics department which led to this project. The author would also like to thank the Rose-Hulman Department of Mathematics for recognizing this work and awarding the author with both the Henry Turner Eddy Award for Applications of Mathematics and the Departmental Award at the Rose Show in 2021 for this research. Finally, the author would like to thank all her friends and family for their support throughout the project, including watching her presentations on several occasions and providing encouragement at every step of the process. The author would especially like to thank her friend Ketu Sayers for suggesting some mathematical tools that helped her solve for the eigenvectors for quartz, as well as for providing some feedback on a later draft of this paper.

\section{References.}

[1] A. Acun, L. Zhang, P. Bampoulis, M. Farmanbar, A. van Houselt, A. N. Rudenko, M. Lingenfelder, G. Brocks, B. Poelsema, M. I. Katsnelson, and H. J. W. ZandVLIET, Germanene: the germanium analogue of graphene, IOP Science, 2015.

[2] R. Band, G. Berkolaiko, and T. K. Weyand, Anomalous Node Count and Singularities in the Dispersion Relation of Honeycomb Graphs, J. Math. Phys., 2015.

[3] G. Berkolaiko And A. Cомech, Symmetry and dirac points in graphene spectrum, Journal of Spectral Theory, (2014).

[4] G. Berkolaiko and P. Kuchment, Introduction to quantum graphs, American Mathematical Society, 2013.

[5] L. CRITChley, Bismuthene - a guide to its properties and potential, AZOMaterials, (2018).

[6] A. Khandelwal, K. Mani, M. H. Karigerasi, and I. Lahiri, Phosphorene - the twodimensional black phosphorous: Properties, synthesis and applications, Materials Science and Engineering, (2017).

[7] M. LiaO, Y. Zang, Z. Guan, H. Li, Y. Gong, K. Zhu, X.-P. Hu, D. Zhang, Y. Xu, Y.Y. Wang, K. He, X.-C. Ma, S.-C. Zhang, And Q.-K. Xue, Superconductivity in few-layer stanene, Nature Physics, 2018.

[8] R. A. Martin, Necessary Conditions on Electric Potential for the Formation of Spectral Touching Points, Baylor University, 2017.

[9] A. Molle, C. Grazianetti, L. Tao, D. Taneja, M. H. Alam, and D. Akinwande, Silicene, silicene derivatives, and their device applications, Royal Society of Chemistry, (2018).

[10] K. Novoselov, Graphene: Materials in the flat-land, Rev. Mod. Phys., 2011. 